\title{
Pheochromocytoma in Italy: a multicentric retrospective study
}

\author{
M Mannelli, L Ianni, A Cilotti, A Conti and the National Study Group on Adrenal Tumors of the Italian Society \\ of Endocrinology \\ Department of Clinical Physiopathology, Endocrine Unit, University of Florence, Viale Pieraccini 6, 50139 Florence, Italy \\ (Correspondence should be addressed to M Mannelli, Dip. Fisiopatologia Clinica, Unità di Endocrinologia, Viale Pieraccini 6, 50139 Firenze, Italy; \\ Email: m.mannelli@dfc.unifi.it)
}

\begin{abstract}
Objective: To conduct an epidemiological study on pheochromocytoma in Italy.

Methods: Data on 284 patients with pheochromocytoma observed between 1978 and 1997 were collected from 18 Italian centers through a questionnaire reporting epidemiological, clinical, laboratory, radiological and surgical data.

Results: $53.6 \%$ of the patients were females and $46.4 \%$ were males. Thirty-two tumors were discovered as incidental adrenal masses. The most frequent referred symptoms were palpitations $(58.1 \%)$, headache $(51.9 \%)$, sweating $(48.8 \%)$ and anxiety $(35.3 \%)$. Their association was present only in $15.5 \%$ of patients. Paroxysmal symptoms were reported in $67.1 \%$ and hypertensive crises in $59.7 \%$ of patients. Normal blood pressure (systolic and diastolic) was present both in the supine and upright positions in $21.1 \%$ of patients. Among laboratory assays, urinary vanylmandelic acid (VMA) was the most widely used $(58.1 \%$ ) and was the least sensitive (25\% of false negative results). Basal plasma catecholamines were found to be normal in $11.3 \%$ of patients but were always elevated when sampled during a hypertensive paroxysm. A clonidine suppression test was performed in 38 patients with no adverse side effects. It gave a false negative response in 2 patients. A glucagon test was performed in 21 patients. It was interrupted for acute hypertension in $52.4 \%$ of patients. Only $5 / 21$ patients were normotensive and had normal basal plasma catecholamines. In these patients the test gave a positive response in four $(80 \%)$. CT $(79.6 \%)$ and I-MIBG scintigraphy $(68.5 \%)$ were the most widely used methods for tumor localization. CT sensitivity was $98.9 \%$ for intra-adrenal and $90.9 \%$ for extraadrenal tumors. MIBG sensitivity was $88.5 \%$. In the 263 patients who underwent surgery, the tumor was intra-adrenal in $89.4 \%$, extra-adrenal in $8.5 \%$, intra- and extra-adrenal in $2.1 \%$, and bilateral in $11.0 \%$ of patients. Malignancy was reported in $9.9 \%$ of cases. Surgery caused remission of hypertension in $59.3 \%$, improvement in $26.8 \%$, and no changes in $13.9 \%$ of patients. In the last group the interval between initial symptoms and diagnosis was significantly longer.

Conclusions: The present study confirms that the clinical presentation of pheochromocytoma is variable and aspecific. Normotension is often present and often the tumor is discovered incidentally. An indication for the routine use of screening methods more sensitive than urinary VMA is strongly suggested. The clonidine test was found to be safe and should be preferred to the glucagon test which has to be restricted to very selected patients. CT and MIBG scintigraphy are almost always successful in localizing the tumor. Reversal of hypertension by surgery seems to depend on an early diagnosis.
\end{abstract}

European Journal of Endocrinology 141 619-624

\section{Introduction}

Pheochromocytoma (pheo) is a rare tumor arising from the chromaffin tissue. It causes hypertension in about $0.1 \%$ of hypertensive patients and presents itself with a highly variable clinical picture $(1-7)$. These two characteristics (rarity and variability) render these tumors very difficult to diagnose so that many of them are discovered incidentally during radiological examinations (especially of the abdomen) or at autopsy $(8,9)$.

Due to the great heterogeneity and the relatively low number of occurrences in many Italian series, in an attempt to standardize the diagnostic and therapeutic management of Italian patients with pheo, the National Italian Study Group on Adrenal Tumors, under the patronage of the Italian Endocrine Society, decided to perform a multicentric retrospective evaluation of patients with pheo.

The study included patients evaluated in 18 Italian centers over the last 20 years.

\section{Patients and methods}

Eighteen centers from all over Italy, belonging either to university departments or to major regional hospitals, participated in the study. 
A questionnaire, previously approved by the participating units was distributed to obtain epidemiological, clinical, laboratory, radiological and surgical data on cases observed between 1978 and 1997.

The questionnaire included personal and familial data of the patients, the referred signs and symptoms, the approximate lag time between initial symptoms and diagnosis, systolic and diastolic blood pressure (BP) and heart rate (HR) in the supine and upright positions and, whenever possible, during a hypertensive crisis, echocardiographic evaluation, basal plasma and/or urinary levels of catecholamines (CA) or metabolites, plasma CA levels during dynamic tests (clonidine or glucagon), localization procedures, pre-surgical pharmacological treatment, surgical findings and blood pressure changes after surgery.

In 263 patients the diagnosis was confirmed at surgery. In the other 21 the presence of a pheo was ascertained by pathological laboratory findings and positive MIBG scintigraphy (10 patients) or positive adrenal sonography, CT or RMI (11 patients).

When analyzing $\mathrm{BP}$, patients were considered normotensive when their systolic BP values were below $140 \mathrm{mmHg}$ and their diastolic BP values were below $90 \mathrm{mmHg}$. Hypertensive systolic records were divided into grade I for values between 140 and $159 \mathrm{mmHg}$, grade II for values between 160 and $179 \mathrm{mmHg}$ and grade III for higher values. Hypertensive diastolic records were divided into grade I for values between 90 and $99 \mathrm{mmHg}$, grade II for values between 100 and $109 \mathrm{mmHg}$ and grade III for higher values.

Measurements of $\mathrm{CA}$ or metabolites, in urine or plasma, were performed using different methods (Table 1). The results were classified as normal or abnormal according to the normality range referred to as normal by each center.

Clonidine tests were performed according to Bravo et al. (10). Plasma CA levels were measured in the supine position before and 120 and $180 \mathrm{~min}$ after oral administration of $300 \mu \mathrm{g}$ clonidine.

In patients with normal basal plasma $\mathrm{CA}$ the response to the test was considered negative when a

Table 1 Percentage distribution of laboratory methods used in the different Centers to measure CA or metabolites.

\begin{tabular}{lccc}
\hline & FL & HPLC & RE \\
\hline urVMA & $52 \%$ & $48 \%$ & - \\
urCA & $58 \%$ & $42 \%$ & - \\
urNE & $15 \%$ & $85 \%$ & - \\
urE & $4 \%$ & $96 \%$ & - \\
pINE & - & $71 \%$ & $29 \%$ \\
pIE & - & $67 \%$ & $33 \%$ \\
\hline
\end{tabular}

FL, fluorimetric assay; HPLC, high pressure liquid chromatography; $R E$, radioenzymatic assay. decrease of at least 50\% was observed (11). In patients with elevated basal plasma CA the test was considered negative when a decrease into the normal range was observed $(10,12)$.

A glucagon test (13) was performed measuring plasma CA before and 1, 3, 5, and $10 \mathrm{~min}$ after i.v. administration of $1 \mathrm{mg}$ glucagon. The response was considered positive when plasma epinephrine and/or plasma norepinephrine increased respectively to more than 2.5 or more than 1.5 times the upper values of their normal range. These criteria were derived from the evaluation of the CA response to glucagon in 60 essential hypertensive patients.

Data were transmitted either on paper support or using the Excel electronic spreadsheet and were collected in our center for the final elaboration.

\section{Statistical analysis}

Data are provided as means \pm S.D. or as means plus range, as appropriate. When the data distribution deviated significantly from a normal one, the geometric mean was computed. Statistical comparisons were performed using either Student's two-tailed $t$-test or chi-square test, as appropriate. Correlations were performed using linear regression analysis. A $P$ value $<0.05$ was considered statistically significant.

\section{Results}

\section{Epidemiological and clinical data}

Data from 284 patients affected by a chromaffin tumor were analyzed. The patient group was composed of $53.6 \%$ female and $46.4 \%$ male patients with a mean age of 44 years (range 8-84 years) for females and 46 years (range 12-79 years) for males. Their body mass indexes were 23.1 (range 12.5-43.7) and 24.2 (range 18-33.0) respectively.

In 258 patients presenting symptoms, the mean interval between initial symptoms and diagnosis was 42 months, ranging from less than 1 month to 30 years.

Familial history for the presence of pheo was positive in $15 \%(39 / 260)$ and negative in $85 \%(221 / 260)$ of cases. When familial, the pheo was due to a MEN 2 syndrome in 30 patients $(77 \%)$, to Von Hippel-Lindau in 4 patients $(10 \%)$ and to neurofibromatosis in 5 patients $(13 \%)$.

The presence of hypertension in first grade relatives of patients was found in $37.1 \%(86 / 232)$ of cases.

The frequency of the different symptoms is reported in Table 2. The most frequent were headache, palpitations, diaphoresis, and anxiety. The association of all these symptoms was found only in $15.5 \%$ of cases while the presence of at least three of them was found in $36.5 \%$ of cases. 
Table 2 Percentage distribution of symptoms presented by 258 patients with pheochromocytoma.

\begin{tabular}{lc}
\hline Symptoms & Distribution (\%) \\
\hline Palpitations & 58.1 \\
Headache & 51.9 \\
Sweating & 48.8 \\
Anxiety & 35.3 \\
Tremors & 25.6 \\
Nausea & 22.4 \\
Dyspnoea & 16.6 \\
Vertigo & 16.5 \\
Abdominal pain & 16.1 \\
Parasthesiae & 11.9 \\
Chest pain & 11.8 \\
Diarrhoea/constipation & 10.6 \\
Fainting & 5.1 \\
Fever & 2.0
\end{tabular}

The presence of symptoms referred as paroxysmal by the patients was reported in $67.1 \%$ of cases $(173 / 258)$.

\section{Cardiovascular picture}

The presence of hypertensive crises in their history was reported by $59.7 \%$ of patients $(154 / 258)$. The absence of hypertension was reported by $28.5 \%(77 / 269)$ and its presence by $71.5 \%(192 / 269)$ of patients. In the group of patients with hypertension $61.5 \%$ classified it as continuous; the others as discontinuous.

Systolic and diastolic BP was measured in the supine position in 249 patients (212 with a sporadic pheo and 37 with a familial pheo) and in the upright position in 156 patients. The distribution of BP values is reported in Table 3. $24.5 \%$ of patients were found to be normotensive in the supine position and $35.3 \%$ in the upright position while $21.1 \%$ of patients were normotensive in both positions. Normotension was more frequent in patients with a familial pheo $(17 / 37 ; 46 \%)$ than in those with a sporadic pheo $(44 / 212 ; 21 \%)(P<0.001)$. Orthostatic hypotension, defined as a fall in systolic BP of at least $30 \mathrm{mmHg}$ or a fall in diastolic BP of at least $20 \mathrm{mmHg}$ was found in $14.7 \%(23 / 156)$ of patients in the first case and $8.3 \%(13 / 156)$ in the second.
HR measured in the supine position (249 patients) was lower than 80 beats/min in $60.5 \%$, between 80 and 100 beats/min in 34.4\% and higher than 100 beats/ min in $5.1 \%$ of patients. HR measured in the upright position (156 patients) was lower than 100 beats/min in $80.8 \%$, between 100 and 110 beats/min in $13.1 \%$ and higher than 110 beats/min in $6.1 \%$ of patients.

$\mathrm{BP}$ was also measured during a hypertensive paroxysm in 101 patients: the mean systolic increase was $72.1 \mathrm{mmHg}$ (range 200-4) and the mean diastolic increase was $32.0 \mathrm{mmHg}$ (range 100-5).

Cardiac evaluation was performed in 110 patients by echocardiography: in $68.2 \%$ cardiac dimensions and functions were normal.

\section{Laboratory results}

In 284 patients the most widely used laboratory methods for the diagnosis of pheo were: urinary vanylmandelic acid (VMA) 58.1\%; total urinary catecholamines (urCA) 30.3\%; urinary norepinephrine (urNE) 46.1\%; urinary epinephrine (urE) 29.9\%; plasma norepinephrine (plNE) $48.9 \%$ and plasma epinephrine (plE) 44.4\%.

Normal values (false negative results) were found in $25 \%$ of VMA assays (41/156), in $14 \%$ of urCA assays $(12 / 86)$, in $21 \%$ of urNE assays $(28 / 131)$, in $34 \%$ of urE assays (29/85), in $17 \%$ of plNE assays (24/139) and in $35 \%$ of plE assays (44/126).

An alteration in either plNE or plE measured in basal conditions was present in $88.7 \%$ of patients (126/ 142). In 11 patients plasma for $C A$ measurement was drawn during a hypertensive paroxysm; a pathological increase in CA concentrations was found in each of them $(100 \%)$, including 4 patients with normal basal plasma CA.

No correlation was found between BP values and basal plasma CA concentrations in 111 patients.

In patients whose basal plNE and plE were both elevated normotension was nonetheless present in 17/66 $(25.7 \%)$. When only one plasma CA was elevated, normotension was more frequent in patients with an Esecreting pheo $(4 / 8=50.0 \%)$ than in patients with an NE-secreting pheo $(3 / 25=12.0 \%)(P<0.0002)$. Among the 12 patients with normal basal plasma $C A, 5$ were normotensive and 7 had grade I hypertension.

Table 3 Percentage distribution of systolic and diastolic values in supine and upright positions in patients with pheochromocytoma before surgery. Normotension and grades of hypertension are defined in the Patients and methods section.

\begin{tabular}{|c|c|c|c|c|}
\hline & Systolic BP supine & Diastolic BP supine & Systolic BP upright & Diastolic BP upright \\
\hline Total number of patients & 249 & 249 & 156 & 156 \\
\hline Normotensive & $31.3 \%$ & $36.5 \%$ & $44.2 \%$ & $45.5 \%$ \\
\hline Hypertension grade I & $28.9 \%$ & $22.9 \%$ & $30.8 \%$ & $24.4 \%$ \\
\hline Hypertension grade II & $18.9 \%$ & $23.3 \%$ & $16.7 \%$ & $17.3 \%$ \\
\hline Hypertension grade III & $20.9 \%$ & $17.3 \%$ & $8.3 \%$ & $12.8 \%$ \\
\hline
\end{tabular}


A clonidine suppression test was performed in 38 patients. Basal plasma CA levels were normal in 7 and elevated in 31 patients.

In the group with normal basal values, clonidine did not suppress plasma NE in $7 / 7$ patients (no false negatives). In the group with elevated basal CA, 20 patients showed abnormal NE and E, 10 patients abnormal NE and normal $\mathrm{E}$ and 1 patient abnormal $\mathrm{E}$ and normal NE. In the 30 patients with abnormal basal NE, clonidine did not suppress plasma NE after 120 and 180 min in 26 patients while it suppressed plasma NE in 1 patient after $120 \mathrm{~min}$, in 1 patient after $180 \mathrm{~min}$ and in 2 patients after 120 and 180 min respectively ( 2 suspicious and 2 false negative responses). In the patient with abnormal basal E clonidine did not suppress plasma $\mathrm{E}$ (no false negative).

A glucagon stimulation test was performed in 21 patients. The test was improperly performed in 7 patients (33.3\%) who showed elevated basal BP values. In the 14 normotensive patients, basal plasma CA was abnormal in 9 patients. The test was interrupted for a hypertensive crisis in 5/7 (71.4\%) hypertensive and in $6 / 14(42.8 \%)$ normotensive patients. In the 5 normotensive patients with normal basal plasma CA the test was interrupted in $1 / 5$ patients $(20 \%)$. It gave a positive response in $4 / 5$ patients $(80 \%)$.

\section{Localization procedures}

The most widely used radiological tool for localization of pheo was CT $(226 / 284 ; 79.6 \%)$. MR was performed in $39 / 284$ patients $(13.7 \%)$ and scintigraphy with I-MIBG in $174 / 254$ patients $(68.5 \%)$ observed after 1983 . The tracer used was ${ }^{131}$ I-MIBG in $66.1 \%$ and ${ }^{123}$ I-MIBG in $33.9 \%$ of cases. The sensitivity of CT was $98.9 \%$ for intra-adrenal pheo and $90.9 \%$ for extra-adrenal pheo. The overall sensitivity of I-MIBG scintigraphy was $88.5 \%(154 / 174)$ with no statistical differences between the two different tracers $\left({ }^{131}\right.$ I-MIBG $87.8 \%$ and ${ }^{123}$ I-MIBG $89.8 \%$ respectively).

In the 263 patients who underwent surgery, the distribution of the localization studies was as follows: abdominal sonography was performed in $69.2 \%$, CT scan in $84.4 \%$, MR in $13.7 \%$, and MIBG scintigraphy in $62.3 \%$ of patients.

Before surgery, localization studies were carried out as follows: 79 (30\%) patients underwent only one radiological procedure (sonography or CT scan or MR); 161 (61.2\%) underwent MIBG scintigraphy and one radiological procedure (among these patients, 144 had a CT scan); $3(1.2 \%)$ underwent only MIBG scintigraphy; in $20(7.6 \%)$ no localization procedures were reported.

\section{Pre-surgery medical treatment}

Medical treatment before surgery was recorded in 170 patients. Alpha-blockers were administered to 106 of them, 82 as monotherapy, the others in association with other drugs. Beta-blockers were given to 27 patients, labetalol to 56, calcium antagonists to 21 and ACE-inhibitors to 6.

\section{Surgical findings}

In the 263 patients who underwent surgery the tumor was intra-adrenal in $89.4 \%$ of patients $(235 / 263)$, extra-adrenal in $8.5 \%(22 / 263)$ and intra- and extraadrenal in $2.1 \%(6 / 263)$. When intra-adrenal, the tumor was bilateral in $11.0 \%(26 / 235)$ and monolateral in $89 \%(209 / 235)$; when monolateral, it was located in the right adrenal in $57 \%$ (134 patients) and in the left adrenal in 32\% (75 patients) $(P<0.0001)$.

Extra-adrenal tumors were more common in patients younger than 20 years $(3 / 17 ; 17.6 \%)$ compared with patients aged 20-60 years (22/187; $11.8 \%)$ and with patients older than 60 years $(3 / 49$; $6.1 \%)$.

Malignancy, as judged by local infiltration or the presence of metastases, was referred in $9.9 \%$ of patients $(26 / 263)$. Malignancy was more frequent in extraadrenal $(9 / 28 ; 32.1 \%)$ than in intra-adrenal pheos $(17 / 235 ; 7.2 \%)$.

Complications during surgery affected $3.4 \%$ of patients.

\section{Blood pressure changes after surgery}

The effects of tumor removal on BP were evaluated in 108 patients who were hypertensive before surgery. After surgery, 64 patients $(59.3 \%)$ became normotensive while $44(40.7 \%)$ remained hypertensive. Nevertheless, in this last group a decrease in BP sufficient to determine a change of hypertensive class was recorded in 26 patients for systolic values and in 25 patients for diastolic values. In 15 patients (13.9\%) no significant decrease in BP was recorded after surgery.

In this last group the time lag between initial symptoms and diagnosis was significantly longer (geometric mean 40.9 months) than in the group of patients who became normotensive after surgery (geometric mean 16.9 months) $(P<0.02)$.

\section{Discussion}

The present retrospective study confirms the wide variability of the clinical picture in patients with pheo and the difficulties in reaching a correct diagnosis. The presence of a paroxysmal event which, although aspecific, has always been considered a hallmark of pheo, has been reported by only $2 / 3$ of the patients.

In agreement with other authors $(2,4,6,7)$, the most frequent symptoms were headache, sweating, palpitation and anxiety but their frequency was rather low and even rarer was the association of three $(36.5 \%)$ or four $(15.5 \%)$ of them. Therefore, in view of the very 
low sensitivity of any symptom or of any association of them, the clinical suspicion is often extremely difficult. This difficulty can explain the long mean time lag (42 months) between initial symptoms and diagnosis and why, quite often, the pheo is discovered as an incidental adrenal mass (32 patients, $11.2 \%$ in the present study) or at autopsy.

Therefore, the most frequent reasons for suspecting a pheo are hypertension, especially if paroxysmal or resistant, and the presence of an adrenal mass, especially if accompanied by hypertension.

It is interesting to point out the increasing incidence of the incidentally discovered pheos. In fact, while $40.6 \%$ were diagnosed in the 15 years between 1978 and 1992 , the majority of them $(59.4 \%)$ were diagnosed in the last 5 years (between 1993 and 1997). Among the patients with an incidentally discovered pheo, 12 (37.5\%) were hypertensive and $20(62.5 \%)$ were normotensive. These data confirm that pheos have to be taken into account in the differential diagnosis of adrenal incidentalomas $(14,15)$ and that the absence of hypertension does not rule out the presence of a pheo. It is likely that in the future an increasing number of pheos will be discovered as an incidental adrenal mass.

In our study $21.1 \%$ of patients were normotensive both in the supine and upright positions. When present, hypertension was mostly mild (Table 2 ) and in $61.5 \%$ of cases it was referred to as discontinuous. These data may, at least in part, explain the finding of normal echocardiographic patterns in $68.2 \%$ of patients.

Hypertensive crises were registered in 101 patients and were characterized by great variability. The mean and peak increase were respectively 72 and $200 \mathrm{mmHg}$ for systolic BP and 32 and $100 \mathrm{mmHg}$ for diastolic BP. Quite surprisingly, no fatal event was reported during paroxysmal hypertension.

The laboratory results show that the most widely used method for the diagnosis of pheo (urinary VMA) is the least sensitive. This finding is in agreement with the data reported in the literature $(5,6,16)$ and should be interpreted as a strong indication to the laboratories for the use of more sensitive assays such as urinary CA or, even better, metanephrines.

Our data confirm that plasma CA values are a very sensitive diagnostic tool when samples are drawn during a hypertensive paroxysm. Outside of this, a normal value does not exclude the presence of a pheo. Recent papers indicate plasma metanephrines as the most sensitive diagnostic index $(17,18)$.

Our data also confirm that the clonidine suppression test is a useful tool in the diagnosis of pheo $(10,19)$. In fact, it gave a false negative response in $2 / 38$ patients. It is worth mentioning that, in the present study, the test gave useful information also in patients with normal basal plasma CA, where it suggested an abnormal regulation of $\mathrm{CA}$ secretion. No adverse reaction was reported during the test.
On the other hand, the glucagon stimulation test must be performed in accurately selected patients because of its potentially dangerous effects. From our data only normotensive patients with normal basal values of CA should be tested with glucagon; all the others should first undergo a clonidine suppression test.

Localization of the tumor relies mainly on CT/MR (20-22). Neverthless, due to the frequency of incidental adrenal masses, MIBG scintigraphy (23, 24) should also be performed before surgery. In fact, in at least 3 patients an association of an extra-adrenal pheo with an incidental adrenocortical mass has been reported.

In the present study, surgical findings are in close agreement with those reported in the literature. In fact, the $9 / 1$ ratio was confirmed for the localization ( intraextra-adrenal), the number (mono- or bilateral), and the biology of the tumor (benign or malignant) (25). In agreement with some authors, the right adrenal gland was significantly more affected than the left one (6).

The effects of surgery on BP are documented by the disappearance of hypertension in about $60 \%$ of patients and by an improvement in about $26 \%$ of patients. BP did not change significantly after surgery in only $14 \%$ of the patients indicating, as suggested by others (26), the presence of other causes of hypertension or nonreversible CA-induced structural changes in the cardiovascular system.

In conclusion, our study confirms the great variability of the clinical picture of pheo. Large numbers of affected patients are normotensive. This finding, besides making the diagnosis very difficult, may explain why many tumors are discovered as incidental adrenal masses.

To confirm the diagnosis the use of one or more sensitive assays (urinary metanephrines, plasma CA, plasma metanephrines) may be necessary. Dynamic tests are seldom required and, while the clonidine suppression test gives useful information without risk for the patient, the use of the potentially dangerous glucagon stimulation test should be restricted to suspected patients who are normotensive and have negative basal measurements.

As already stated, presurgical localization relies on CT and MIBG scintigraphy.

As a final remark, surgery cures hypertension in 60\% and ameliorates it in $26 \%$ of the patients. Delay in the diagnosis seems to be responsible for the irreversibility of hypertension after surgery.

\section{Acknowledgements}

The participants in the National Study Group on Adrenal Tumors of the Italian Society of Endocrinology were as follows: A Angeli, A Ali, M Terzolo (Torino); G Favia, F Lumachi (Padova); B Ambrosi, E Passini (Milano); C Letizia (Roma); G Angeletti, P Ferolla, F Santeusanio (Perugia); P Bernante, G Opocher, 
C Scaroni, B Vianello (Padova); M Doga, A Giustina (Brescia); G Delitala, M Palermo (Sassari); L Antonio, C Battista, T Libuse, R Rossi (Napoli); G P Bernini, A Monti (Pisa); M R Ambrosio, M Campo, E Degli Uberti (Ferrara); C Invitti (Milano); G B Contessi, G Francia, A Orsolini(Verona); M R Ghiggi, M Torlontano (S Giovanni Rotondo); G Arnaldi, F Mantero, A Taccariti (Ancona); G Covi, P Delva, P Minuz (Verona); F Camanni, E Mazza (Torino).

\section{References}

1 Manger WM \& Gifford RW. Pheochromocytoma. New York: Springer-Verlag, 1977.

2 Sever PS, Roberts JC \& Snell ME. Phaeochromocytoma. Clinics in Endocrinology and Metabolism 19809 543-568.

3 Bravo EL \& Gifford RW Jr. Pheochromocytoma: diagnosis, localization and management. New England Journal of Medicine 1984311 1298-1303.

4 Cryer PE. Phaeochromocytoma. Clinics in Endocrinology and Metabolism 198514 203-220.

5 Plouin PF, Chatellier G, Rougeot MA, Duclos JM, Pagny JY, Corvol Pet al. Recent developments in pheochromocytoma diagnosis and imaging. Advances in Nephrology from the Necker Hospital 198817 275-286.

6 Fonseca V \& Bouloux PM. Phaeochromocytoma and paraganglioma. Baillieres Clinical Endocrinology and Metabolism 1993 7 509-544.

7 Young WF Jr. Pheochromocytoma and primary aldosteronism: diagnostic approaches. Endocrinology and Metabolism Clinics of North America 199726 801-827.

8 Bittar DA. Unsuspected phaeochromocytoma. Canadian Anaesthetists' Society Journal 198229 183-184.

9 Stenstroem G \& Svardsudd K. Pheochromocytoma in Sweden 1958-1981. An analysis of the National Cancer Registry Data. Acta Medica Scandinavica $1986220225-232$.

10 Bravo EL, Tarazi RC. Fouad FM, Vidt DG \& Gifford RWJ. Clonidine suppression test: a useful aid in the diagnosis of pheochromocytoma. New England Journal of Medicine 1981305 623-626.

11 Elliott WJ \& Murphy MB. Reduced specificity of the clonidine suppression test in patients with normal plasma catecholamine levels. American Journal of Medicine 1988 84 419-424.

12 Grossman E, Goldstein DS, Hoffman A \& Keiser HR. Glucagon and clonidine testing in the diagnosis of pheochromocytoma. Hypertension 199117 733-741.
13 Lawrance AM. Glucagon provocative test for pheochromocytoma. Annals of Internal Medicine 196766 1091-1096.

14 Latronico AC \& Chrousos GP. Adrenocortical tumors. Journal of Clinical Endocrinology and Metabolism 199782 1317-1324.

15 Mantero F, Masini AM, Opocher G, Giovagnetti M \& Arnaldi G. Adrenal incidentaloma: an overview of hormonal data from the National Italian Study Group. Hormone Research 199747 284-289.

16 Bravo EL, Tarazi RC, Gifford RW \& Stewart BH. Circulating and urinary catecholamines in pheochromocytoma. New England Journal of Medicine $1979301682-686$.

17 Lenders JW, Keiser HR, Goldstein DS, Willemsen JJ, Friberg P \& Jacobs MC. Plasma metanephrines in the diagnosis of pheochromocytoma. Annals of Internal Medicine 1995123 101-109.

18 Eisenhofer G, Keiser H, Friberg P, Mezey E, Huynh TT, Hiremalagur B et al. Plasma metanephrines are markers of pheochromocytoma produced by catechol-O-methyltransferase within tumors. Journal of Clinical Endocrinology and Metabolism $1998832175-2185$.

19 Mannelli M, De Feo ML, Maggi M, Pupilli C, Opocher G, Valenza T et al. Usefulness of basal catecholamine plasma levels and clonidine suppression test in the diagnosis of pheochromocytoma. Journal of Endocrinological Investigation 1987 10 377-382.

20 Welch TJ, Sheedy PF, Van Heerden JA, Sheps SG, Hattery RR \& Stephens DM. Pheochromocytoma: value of computed tomography. Radiology $1983184501-503$.

21 Francis IR, Glazer GM, Shapiro B, Sisson IC \& Gross BH. Complementary roles of $\mathrm{CT}$ and $\mathrm{I}^{131}$-MIBG scintigraphy in diagnosing pheochromocytoma. American Journal of Roentgenology $1983141719-725$.

22 Schmedtje JFJ, Sax S, Pool JL, Goldfarb RA \& Nelson EB. Localization of ectopic phaeochromocytomas by magnetic resonance imaging. American Journal of Medicine 198783 770-772.

23 Sisson JC, Frager MS, Valk TW, Gross MD, Swanson DP, Wieland DM et al. Scintigraphic localization of phaeochromocytoma. New England Journal of Medicine 1981305 12-17.

24 Shapiro B. Imaging of catecholamine-secreting tumours: uses of MIBG in diagnosis and treatment. Baillieres Clinical Endocrinology and Metabolism 19937 491-507.

25 Manger WM \& Gifford RWJ. Diagnosis. In Clinical and Experimental Pheochromocytoma. Cambridge, Massachusetts: Blackwell Science, 1996.

26 Plouin PF, Chatellier G, Fofol I \& Corvol P. Tumor recurrence and hypertension persistence after successful pheochromocytoma operation. Hypertension 199729 1133-1139.

Received 6 April 1999

Accepted 18 August 1999 